\title{
CHARACTERIZATION OF A FAMILY OF SIMPLE GROUPS BY THEIR CHARACTER TABLE, II
}

\author{
MARCEL HERZOG AND DAVID WRIGHT
}

(Received 3 December 1979)

Communicated by D. E. Taylor

\begin{abstract}
It is shown that the simple groups $G_{2}(q), q=3^{f}$, are characterized by their character table. This result completes characterization of the simple groups $G_{2}(q), q$ odd, by their character table.
\end{abstract}

1980 Mathematics subject classification (Amer. Math. Soc.): 20 D 08. 20 C 15.

The aim of this paper is to prove the following result:

THEOREM 1. The character table of $G_{2}(q)$, q odd, determines $G_{2}(q)$.

By Theorem 3.2 in Herzog and Wright (1977), it suffices to deal with the case $q=3^{f}$. Thus we prove, using the character tables of $G_{2}\left(3^{f}\right)$ recently computed by Enomoto (1976), that the following theorem holds:

THEOREM 2. The character table of $G_{2}(q), q=3^{f}$, determines $G_{2}(q)$.

Proof. We shall use the notation of Enomoto (1976) for elements and characters of $G_{2}(q), q=3^{f}$. In addition, we shall denote by $\operatorname{Irr}(G)$ the set of irreducible characters of $G$ and if $x \in G, \mathrm{Cl}(x)$ denotes the conjugacy class of $x$ in $G$.

Suppose that ${ }^{*} G$ is a group with the same character table as $G_{2}(q), q=3^{f}$. Put an asterisk in front of each conjugacy class representative, character, and so on, of ${ }^{*} G$, to distinguish it from the same in $G_{2}(q)$. Since a character table determines the order of the group and the lattice of normal subgroups, see Feit (1967), ${ }^{*} G$ is simple with ${ }^{*} G \mid=q^{6}\left(q^{2}-1\right)\left(q^{6}-1\right)$. The first step is to establish that ${ }^{*} G$ has a unique conjugacy class of involutions.

Lemma 3. The only conjugacy class of involutions in ${ }^{*} G$ is that represented $b y{ }^{*} B_{1}$. 
Proof. By Enomoto(1976), p. $239,{ }^{*} B_{1}$ is the only class representative with the full 2-power of $\left|{ }^{*} G\right|$ dividing the order of its centralizer. Hence ${ }^{*} B_{1}$ is an involution. The classes of $G_{2}(q)$ denoted by $A_{i}$ or $A_{i j}$ in Enomoto (1976) consist of 3-elements, and by Lambert (1972), Property 2.5, also ${ }^{*} A_{i}$ and ${ }^{*} A_{i j}$ are 3 -elements. Let ${ }^{*} F$ be a conjugacy class representative in ${ }^{*} G,{ }^{*} F \neq{ }^{*} A_{i},{ }^{*} A_{i j},{ }^{*} B_{1}$. Then by Enomoto (1976), p. 239 ,

$$
\left|C_{*_{G}}\left({ }^{*} F\right)\right| \leqslant q(q+1)\left(q^{2}-1\right)
$$

hence

$$
\left|C 1_{* G}\left({ }^{*} F\right)\right| \geqslant|G| \cdot q(q+1)\left(q^{2}-1\right)=q^{5}\left(q^{5}-q^{4}+q^{3}-q^{2}+q-1\right) .
$$

Consequently, we get

$$
\left|\mathrm{C} 1 *_{G}\left({ }^{*} F\right)\right| \geqslant q^{10}-q^{9} .
$$

Suppose that ${ }^{*} F$ is an involution. If $t$ is the number of involutions in ${ }^{*} G$, then by Feit (1967), p. 23,

$$
t+1 \leqslant \sum^{*} Y_{i}(1)=\sum Y_{i}(1)
$$

where ${ }^{*} Y_{i}\left(Y_{i}\right)$ runs through $\operatorname{Irr}\left({ }^{*} G\right)\left(\operatorname{Irr}\left(G_{2}(q)\right)\right.$. To obtain an upper bound for $\sum Y_{i}(1)$ the following inequalities were used:

$$
\begin{aligned}
& q \geqslant 3, \quad(q+1)^{2} \leqslant 2 q^{2}, \quad q^{i}+1 \leqslant 4 q^{i} / 3, \quad i=1, \ldots, \\
& q^{i}-d \leqslant q^{i} \quad \text { for } d \geqslant 0, \quad\left(q^{i}\right)^{2}+q^{i}+1 \leqslant 3 q^{2 i} / 2, \quad i=1, \ldots,
\end{aligned}
$$

and

$$
\left(q^{i}\right)^{2}-q^{i}+1 \leqslant q^{2 i}, \quad i=1, \ldots .
$$

We get. using the notation of Enomoto (1976):

$$
\sum_{i=0}^{12} \theta_{i}(1) \leqslant 1+\frac{3}{2} q^{4}+6 \frac{13}{18} q^{5}+2 \frac{1}{2} q^{6} \leqslant 1+5 q^{6}
$$

and

$$
\sum_{i=1}^{14} r\left(X_{i}\right) X_{i}(1) \leqslant 3 q^{6}+3 q^{7}+1 \frac{35}{108} q^{8} \leqslant 3 q^{8},
$$

where $r\left(X_{i}\right)$ is the number of characters of type $X_{i}$ and degree $X_{i}(1)$ in $\operatorname{Irr}\left(G_{2}(q)\right)$. Thus:

$$
\sum Y_{i}(1) \leqslant 1+4 q^{8} \leqslant 1+4 q^{9} / 3 .
$$

If ${ }^{*} F$ were an involution, we would get from (1), (2) and (3) that

$$
1+q^{10}-q^{9} \leqslant 1+4 q^{9} / 3
$$

hence $q \leqslant 7 / 3$, a contradiction. Thus ${ }^{*} F$ is not an involution, proving the lemma. 
We need also:

LEMMA 4. 2-rank $* G \leqslant 3$.

Proof. By Lemma 3 and by Lemma 2.1(b) in Herzog and Wright (1977), it suffices to find an $X \in \operatorname{Irr}\left(G_{2}(q)\right)$ such that

$$
X\left(A_{1}\right)-X\left(B_{1}\right) \not \equiv 0 \quad(\bmod 16) .
$$

First suppose that $q=3^{f}, f$ odd. Then $q \equiv 3$ or 11 (mod 16) and we get, using the tables of Ecomoto (1976):

$$
3\left(\theta_{3}\left(A_{1}\right)-\theta_{3}\left(B_{1}\right)\right)=q\left(q^{4}+q^{2}-2\right) \equiv 8 \quad(\bmod 16) .
$$

Hence $\theta_{3}$ satisfies $(4)$ in this case. For $q=3^{f}, f$ even, we have: $q \equiv 1$ or $9(\bmod 16)$. Consider $X_{1}(k), k \in{ }^{2} R_{2}$. Clearly $1,2 \in{ }^{2} R_{2}$, hence:

$$
\left(X_{1}(1)\left(A_{1}\right)-X_{1}(1)\left(B_{1}\right)\right)-\left(X_{1}(2)\left(A_{1}\right)-X_{1}(2)\left(B_{1}\right)\right)=2(q+1)^{2} \equiv 8 \quad(\bmod 16)
$$

and either $X_{1}(1)$ or $X_{1}(2)$ satisfies (4). The lemma is proved in all cases.

We now complete the proof of Theorem 2 . By Lemma 42 -rank ${ }^{*} G \leqslant 3$. Since $G_{2}(q)$ has 2-rank 3, by Lemma 2.1.(b) in Herzog and Wright (1977) $X\left(A_{1}\right)-X\left(B_{1}\right) \equiv 0(\bmod 8)$ for each $X \in \operatorname{Irr}\left(G_{2}(q)\right)$. Consequently, Corollary 2.5 in Herzog and Wright (1977) and the Note following it yield: 2 -rank ${ }^{*} G=3$. As in Herzog and Wright (1977), p. 303, we conclude, using Stroth's (1976) classification of simple groups of 2-rank 3 , that ${ }^{*} G=G_{2}(q)$ unless $q=3$. In the latter case ${ }^{*} G \mid=3^{6} \cdot 8\left(3^{6}-1\right)$ and it is easy to check that the only group of that order in Stroth's list is $G_{2}(3)$. Hence ${ }^{*} G=G_{2}(q)$ for each $q=3^{f}$, thus proving Theorem 2 .

\section{References}

Hikoe Enomoto (1976). 'The characters of the finite Chevally group $G_{2}(q), q=3^{f^{\prime}}$, Japan J. Math. 2. 19l-248.

Walter Feit (1967), Characters of finite groups (Mathematics Lecture Notes, W. A. Benjamin Inc.. New York).

Marcel Herzog and David Wright (1977), 'Characterization of a family of simple groups by their character table', J. Austral. Math. Soc. Ser. A 24, 296-304.

P. J. Lambert (1972), 'Characterizing groups by their character tables, I. Quart. J. Math. Oxford Ser., 23, $427-433$.

G. Stroth (1976), 'Über Gruppen mit 2-Sylow-Durchschnitten von Rang $\leqq 3$ ' I and II, J. Algebra 43. 398-456 and 457-505.

\section{Department of Mathematics}

Tel-Aviv University

Tel-Aviv

Israel 